\title{
Breit-Wigner width for two interacting particles \\ in one-dimensional random potential
}

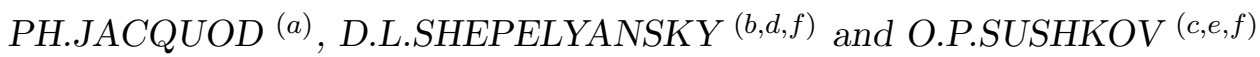 \\ (a) Institut de Physique, Université de Neuchâtel, \\ 1, Rue A.L. Breguet, 2000 Neuchâtel, Suisse \\ (b) Institute for Theoretical Physics, University of California \\ Santa Barbara, CA 93106-4030 \\ (c) Institute for Theoretical Atomic and Molecular Physics, \\ Harvard-Smithsonian Center for Astrophysics, MS14, \\ 60 Garden Street, Cambridge, Massachusetts 02138
}

\begin{abstract}
For two interacting particles (TIP) in one-dimensional random potential the dependence of the Breit-Wigner width $\Gamma$, the local density of states and the TIP localization length on system parameters is determined analytically. The theoretical predictions for $\Gamma$ are confirmed by numerical simulations.
\end{abstract}

PACS. 72.15Rn, 71.30+h 
Recently, the problem of two interacting particles (TIP) in a random potential has attracted interest of different groups [1, 2, 3, 4, 6, 7]. It has been shown that two repulsive/attracting particles can propagate together on a distance $l_{c}$ much larger than one-particle localization length $l_{1}$ in absence of interaction. The first analytical studies [1, 2] for TIP with on site interaction on a one-dimensional one channel lattice gave the following estimate $l_{c} / l_{1} \sim \Gamma \rho \sim(U / V)^{2} l_{1}$, where $U$ is strength of the interaction, $V$ is intersite hopping matrix element, $\rho \sim l_{1}^{2} / V$ is density of the two-particle states coupled by the interaction, and $\Gamma \sim U^{2} / V l_{1}$ is the interaction induced transition rate between these states. The numerical investigations [3, 4] definitely confirmed existence of the strong enhancement of $l_{c}$ due to interaction. However, a direct verification of the above estimate is quite difficult even for the modern computer facilities due to the strong increase of required basis with $l_{1}$. Also the recent numerical results of von Oppen et. al. [4] and Weinmann and Pichard [5] indicate in one-dimensional case almost linear growth of the enhancement factor for $l_{c}$ with $U$ instead of expected $U^{2}$. Due to all these things it would be important to have a more rigorous derivation of the factor $l_{c} / l_{1}$ for this on a first glance quite simple problem, at least in a one-dimensional case. To reach this aim we started from the computation of the rate $\Gamma$ which also characterizes the spread width of the Breit-Wigner distribution for eigenfunctions in the basis of eigenstates of noninteracting particles [8, 9, 10]. If the parameter dependence of $\Gamma$ is known then the ratio $l_{c} / l_{1}$ can be determined from the relation $l_{c} / l_{1} \sim \Gamma \rho$ which have been checked in models of superimposed band random matrices [1, 8, 9, 10. In the present work for calculation of $\Gamma$ we use the technique developed in [11] which allows to account all orders in the interaction. 
We consider one dimensional Hubbard model with Hamiltonian

$$
H=-V \sum_{n \sigma}\left(a_{n+1 \sigma}^{\dagger} a_{n \sigma}+a_{n \sigma}^{\dagger} a_{n+1 \sigma}\right)+U \sum_{n} a_{n \uparrow}^{\dagger} a_{n \downarrow}^{\dagger} a_{n \downarrow} a_{n \uparrow}
$$

Here $a_{n}^{\dagger}$ is a creation operator of the particle at the site $n, V$ is hopping matrix element, and $U$ is on site interaction. We assume that particles are distinguishable and denote the type of particle by spin $\sigma= \pm 1 / 2$. Single particle eigenstate is plane wave $|p\rangle=\frac{1}{\sqrt{L}} e^{i p n}$ with dispersion $\epsilon_{p}=-2 V \cos p,-\pi \leq p \leq \pi$. We set lattice spacing equal to unity. The size of the lattice is denoted by $L$.

The Breit-Wigner width can be found in the following way. Forward scattering amplitude $f$ for particles with different spins is given by series of diagrams presented at Fig.1. Solid line represents a particle, and wavy line is matrix element of the interaction $\left\langle p_{3} p_{4}|\hat{U}| p_{1} p_{2}\right\rangle=\frac{U}{L} \delta_{p_{1}+p_{2}, p_{3}+p_{4}}$. Due to optical theorem width of the state $\left|p_{1} p_{2}\right\rangle=\left|p_{1}\right\rangle\left|p_{2}\right\rangle$ is related to the forward scattering amplitude:

$$
\Gamma / 2=-\operatorname{Im} f
$$

One can easily check the coefficient in this relation considering diagram Fig. 1b which gives usual Fermi golden rule:

$$
\begin{aligned}
\Gamma \approx-2 I m f_{1 b} & =-2 \operatorname{Im} \sum_{p_{3} p_{4}} \frac{\left|\left\langle p_{3} p_{4}|\hat{U}| p_{1} p_{2}\right\rangle\right|^{2}}{E-\epsilon_{3}-\epsilon_{4}+i 0}= \\
& =2 \pi \sum_{p_{3} p_{4}}\left|\left\langle p_{3} p_{4}|\hat{U}| p_{1} p_{2}\right\rangle\right|^{2} \delta\left(E-\epsilon_{3}-\epsilon_{4}\right) .
\end{aligned}
$$

Here $E$ is energy of the initial state $E=\epsilon_{1}+\epsilon_{2}$.

Born term in the amplitude $f$ is given by Fig. 1a and equals $f_{1 a}=U / L$. Calculation of the diagram Fig. 1b is also straightforward

$$
f_{1 b}=\sum_{p_{3} p_{4}} \frac{\left|\left\langle p_{3} p_{4}|\hat{U}| p_{1} p_{2}\right\rangle\right|^{2}}{E-\epsilon_{3}-\epsilon_{4}+i 0}=\frac{U^{2}}{L^{2}} \sum_{p_{3}} \frac{1}{E+2 V \cos p_{3}+2 V \cos \left(p-p_{3}\right)}=
$$




$$
=\frac{U^{2}}{L^{2}} \int_{-\pi}^{\pi} \frac{L d p_{3} / 2 \pi}{\left[E+2 V \cos p_{3}+2 V \cos \left(p-p_{3}\right)\right]}=\frac{U^{2} / L}{\sqrt{E^{2}-16 V^{2} \cos ^{2} p / 2}}
$$

where $p=p_{1}+p_{2}=p_{3}+p_{4}$ is total quasi-momentum. Higher orders in Fig. 1 correspond to simple iterations of the box Fig. 1b. Therefore summation of the ladder is reduced to geometrical progression and the result is

$$
f(E, p)=\frac{U / L}{1-U / \sqrt{E^{2}-16 V^{2} \cos ^{2} p / 2}} .
$$

The scattering amplitude depends only on total energy $-4 V \leq E \leq 4 V$ and total momentum $-\pi \leq p \leq \pi$. The branch of square root should be chosen in such a way that $\operatorname{Im} f \leq 0$.

With amplitude (可) one can easily calculate the Breit-Wigner width using optical theorem (2). But we are interested in the average width at given energy. So we have to average over momentum $p$. Density of the two particle states is of the form

$$
\begin{aligned}
\rho(E, p) & =\int_{-\pi}^{\pi} \frac{L d p_{1}}{2 \pi} \int_{-\pi}^{\pi} \frac{L d p_{2}}{2 \pi} \delta\left(p-p_{1}-p_{2}\right) \delta\left(E+2 V \cos p_{1}+2 V \cos p_{2}\right)= \\
& =\frac{L^{2} /\left(8 \pi^{2} V\right)}{\sqrt{\cos ^{2} p / 2-E^{2} / 16 V^{2}}} .
\end{aligned}
$$

It is nonzero only if square root is real. After integration over momenta we find

$$
\rho(E)=\int_{-\pi}^{\pi} \rho(E, p) \frac{d p}{2 \pi} \approx \frac{L^{2}}{2 \pi^{2} V}\left(\ln \frac{16 V}{|E|}+0.18 \frac{|E|}{4 V}\right)
$$

The integral in (đ) can not be exactly expressed in terms of elementary functions. Presented approximate formula is valid with accuracy better than $1 \%$ in the interval $-4 V \leq$ $E \leq 4 V$. Now we can find the average Breit-Wigner width.

$$
\begin{aligned}
\Gamma(E) & =-2 \operatorname{Im} \int f(E, p) \rho(E, p) \frac{d p}{2 \pi} / \rho(E)= \\
& =\frac{8 V u^{2} / L}{(\ln 4 / \epsilon+0.18 \epsilon) \sqrt{\left|u^{2}-\epsilon^{2}\right|\left(1+u^{2}-\epsilon^{2}\right)}} \cdot F(Z) .
\end{aligned}
$$


Here $u=|U / 4 V|$ and $\epsilon=|E / 4 V|$ is interaction and energy expressed in units of band width: $0 \leq \epsilon \leq 1$. The function $F(Z)$ is defined by

$$
\begin{aligned}
F(Z) & =\left\{\begin{array}{l}
\arctan Z, \quad \text { for } \mathrm{u} \geq \epsilon \\
\frac{1}{2} \ln \frac{1+Z}{1-Z}, \quad \text { for } \mathrm{u} \leq \epsilon
\end{array}\right. \\
Z & =\sqrt{\frac{\left|u^{2}-\epsilon^{2}\right|\left(1-\epsilon^{2}\right)}{\epsilon^{2}\left(1+u^{2}-\epsilon^{2}\right)}} .
\end{aligned}
$$

At small energy $\left(\epsilon^{2} \ll u^{2}, 1\right)$ formula (8) can be substantially simplified

$$
\Gamma \approx \frac{4 \pi V}{L} \cdot \frac{1}{\ln 4 / \epsilon} \cdot \frac{u}{\sqrt{1+u^{2}}}
$$

so that at small interaction $\left(\epsilon^{2} \ll u^{2} \ll 1\right)$ it is linear in the interaction. In other limit $\left(u^{2} \ll \epsilon^{2},\left(1-\epsilon^{2}\right)\right)$ the width (8) is quadratic in the interaction with logarithmic correction:

$$
\Gamma \approx \frac{8 V}{L} \cdot \frac{1}{(\ln 4 / \epsilon+0.18 \epsilon)} \cdot \frac{u^{2}}{\epsilon} \ln \frac{2 \epsilon \sqrt{1-\epsilon^{2}}}{u}
$$

The value of $\Gamma$ in (10) is significantly larger than in (11) due to the growth of two-particle density of states (7) near the center of the band.

If we now add to the Hamiltonian (11) a single particle random potential $H_{\text {rand }}=$ $\sum w_{n} a_{n \sigma}^{\dagger} a_{n \sigma}$ with a disorder homogeneously distributed in the interval $-W \leq w_{n} \leq W$, then one particle eigenstates in infinite lattice become localized with localization length $l_{1} \approx 24(V / W)^{2} \sqrt{1-\epsilon_{1}^{2} / 4 V^{2}}$, where $\epsilon_{1}$ is one particle energy. However as soon as $l_{1} \gg 1$ the above calculation of the average width remains valid. The reason for this is that $l_{1} \gg 1$ is the only condition which we need to formulate scattering problem and to use conventional diagram technique. Distribution of $\Gamma$ depends on the relation between size of the box $L$ and the localization length $l_{1}$. If $L \leq l_{1}$ all values of $\Gamma$ are of the order 
of the average value given by (8). For $L \gg l_{1}$ the average value is still given by (8). However in this case $\Gamma$ vanishes for majority of the states. These are the states in which particles are localized far from each other and practically do not interact. On other hand the width for the states with interparticle distance of the order $l_{1}$ is approximately the same as for particles in a box of size $L \approx l_{1}$ so that $\Gamma$ is given by eqs.(8),(9) with $L$ replaced by $l_{1}$. The two-particle localization length $l_{c}$ for such states is determined by the relation $l_{c} / l_{1} \sim \Gamma(E) \rho(E)$, with $\Gamma$ calculated at $L \sim l_{1}$. This relation is valid if many unperturbed states are mixed by interaction [1], 2] so that $\Gamma(E) \rho(E)>1$. In the opposite case $\Gamma(E) \rho(E) \ll 1$ the above relation is not valid [8, 9, 10] and the interaction can be treated in a perturbative way. In this regime "Rabi oscillations" between two quasi-degenerate levels play an important role [5].

Above we have considered distinguishable particles. The generalization to identical particles is rather simple: the width $\Gamma$ vanishes if coordinate wave function is antisymmetric, and it is doubled in comparison with eqs.(8), (9) if coordinate wave function is symmetric.

To check the above theoretical formula for the Breit-Wigner width $\Gamma$ we studied numerically the model (11) of two identical interacting particles (symmetric coordinate wave function) in the disordered potential on a ring of size $L$ which is less or comparable with one-particle localization length $l_{1} \approx 24(V / W)^{2}$. Using Lanczos technique (see for example [12]) we determined the local density of states for symmetric configurations in the basis of noninteracting eigenstates:

$$
\rho_{W}\left(E-\epsilon_{m_{1}}-\epsilon_{m_{2}}\right)=\sum_{\lambda}\left|\psi_{\lambda}\left(m_{1}, m_{2}\right)\right|^{2} \delta\left(E-E_{\lambda}\right)
$$


Here $E_{\lambda}$ is the eigenenergy of TIP while $\epsilon_{m_{1,2}}$ are one-particle eigenenergies. The dependence of $\rho_{W}$ on $E$ is well described by the Breit-Wigner distribution

$$
\rho_{W}(E)=\frac{\Gamma}{2 \pi\left[E^{2}+\Gamma^{2} / 4\right]}
$$

an example of which is shown in Fig.2 . The comparison of numerically obtained $\Gamma$ with theoretical prediction (8), (9) in the regime $\Gamma(E) \rho(E)>1$ is shown in Figs.3,4 for different energies as the function of interaction. The theory gives good agreement with numerical results for $15 \leq L \leq 300$ and variation of scaled width $\Gamma L / V$ by more than 2 orders of magnitude. For the states with the energy close to the band center $(E \approx 0)$ (Fig.3) the dependence of $\Gamma \rho$ on $U$ is almost linear for $U<V$ (see (7), (10)). Therefore, the TIP localization length $l_{c}$ according to the relation $l_{c} / l_{1}=C \Gamma \rho \approx 2 C l_{1}(U / V) / \pi$ also varies linearly with $U$. Here, we took the values of $\Gamma$ and $\rho$ at $L=l_{1}$ and introduced the numerical coefficient $C$ to take into account the uncertainty of this choice. According to the numerical results [4] at the center of the band $l_{c} / l_{1} \approx 0.2 l_{1}(U / V)$ which is in good agreement with the above theoretical expression and gives $C \approx 1 / 4$.

For energies away from the band center and small interaction $|U| \ll|E|$ the enhancement factor according to (7), (11) is $l_{c} / l_{1} \approx l_{1} U^{2} \ln (2 E / U) /\left(4 \pi^{2} V E\right)$ where we have used the above value of $C$. The dependence on $U$ is almost quadratic in agreement with the first estimate [1], 2]. However, due to the logarithmic correction, to observe clearly the $U^{2}$ behavior one should go to really small $U$ values and since the condition $\Gamma \rho>1$ should be also satisfied this can be reached only for quite large values of $l_{1}$ or $L$. In this respect our numerical approach based on the measurement of $\Gamma$ is more efficient than the one used in [4]. It allows to see the behavior $U^{2} \ln U$ away from the band center in agreement with 
the theory (8), (9) (see insert in Fig.4). At moderate $U / V>0.3$ values in the presence of numerical fluctuations the dependence of $\Gamma$ on $U$ is hardly distinguishable from a linear one (see normal scale in Fig.4). In our opinion this is the reason why the linear behavior in $U$ had been attributed in [⿶] also to the states away from the band center. As for the result of Ref. [5] the system size there was too small $(L=25)$ and the main part of the data (Fig. 4 with $U / V<0.4$ ) corresponds to the different regime $\Gamma \rho<1$. In this perturbative case the typical energy scale which determines the change in level statistics is determined by Rabi oscillation frequency in a pair of quasi-degenerate states which is proportional to $U$ [5]. Also, one should keep in mind that the results there are integrated over the whole energy band including the center of the band where the dependence on $U$ is linear even for $\Gamma \rho>1$.

Turning back to our numerical data (Fig. 4) we would like to mention that there is a significant difference from the theory for negative $U<-1$.. Generally, we should expect such difference for $|U| \gg 1$ when the spectrum is composed from two separated energy bands and the basis of plane waves used for computation of width $\Gamma$ becomes inadequate. For example, in this regime the density of states is not described by (7). However, we cannot say why this change goes in so asymmetric way for negative and positive $U$ while for $|U|<1$ the width $\Gamma$ is independent of $\operatorname{sign} U$ in agreement with the theory. We would like to note that such asymmetry for attraction and repulsion away from the band center and relatively strong interaction $U \approx V$ has been seen recently in [⿶] for the ratio $l_{c} / l_{1}$. Also a change in the behavior of $\Gamma$ has been observed in [5] for $U>V$.

In summary, taking diagrammatically into account the effects of interaction we have 
derived the analytical formula for the Breit-Wigner width $\Gamma$ which determines the enhancement factor $l_{c} / l_{1} \sim \Gamma \rho>1$ for TIP in one-dimensional random potential. Our analytical and numerical approaches can be also used for calculation of the TIP width in 2- and 3-dimensional disordered systems where according to Imry estimate [2] interaction between two quasi-particles can strongly affect transport properties.

We acknowledge fruitful discussions with V.V. Flambaum, M.Yu. Kuchiev and P.G.Silvestrov. We also thank the Centro Svizzero di Calcolo Scientifico for allocation of CPU time on their NEC SX-3. One of us (OPS) thanks Laboratoire de Physique Quantique, Université Paul Sabatier for hospitality and financial support during the initial stage of this work. This research was supported in part by the National Science Foundation under Grant No. PHY94-07194, NSF through a grant for the Institute for Theoretical Atomic and Molecular Physics at Harvard University and the Smithsonian Astrophysical Observatory and the Fonds National Suisse de la Recherche. 


\section{References}

[d] On leave from Laboratoire de Physique Quantique, UMR C5626 du CNRS, Université Paul Sabatier, 31062 Toulouse Cedex, France

[e] On leave from School of Physics, The University of New South Wales, Sydney 2052, Australia

[f] Also : Budker Institute of Nuclear Physics, 630090 Novosibirsk, Russia

[1] D.L. Shepelyansky, Phys. Rev. Lett. 73, 2607 (1994).

[2] Y. Imry, Europhys. Lett 30, 405 (1995).

[3] K. Frahm, A. Müller-Groeling, J.-L. Pichard and D. Weinmann, Europhys. Lett. 31, 405 (1995); D. Weinmann, A. Müller-Groeling, J.-L. Pichard and K. Frahm, Phys. Rev. Lett. 75, 1598 (1995).

[4] F. von Oppen, T. Wettig and J. Müller, Phys. Rev. Lett. 76, 491 (1996); F. von Oppen and T. Wettig, Proc. Int. Conf. "Correlated fermions and transport in mesoscopic systems", XXXIst Rencontres de Moriond (1996).

[5] D. Weinmann and J.-L. Pichard, Phys. Rev. Lett. 77, 1556 (1996).

[6] F. Borgonovi and D.L. Shepelyansky, Nonlinearity 8, 877 (1995); J. de Phys. I France 6, 287 (1996).

[7] K. Frahm, A. Müller-Groeling and J.-L. Pichard, Phys. Rev. Lett. 76, 1509 (1996).

[8] P. Jacquod and D. L. Shepelyansky, Phys. Rev. Lett. 75, 3501 (1995).

[9] Y. V. Fyodorov and A. D. Mirlin, Phys. Rev. B 52, R11580 (1995). 
[10] K. Frahm and A. Müller-Groeling, Europhys. Lett. 32, 385 (1995).

[11] O.P.Sushkov, unpublished (1996).

[12] E.Dagotto, Rev. Mod. Phys. 66, 763 (1994). 


\section{Figure captions}

Fig. 1: Diagrams for the forward scattering amplitude $f$ in (2) - (5).

Fig. 2: Local spectral density $\rho_{W}(E)$ computed for the TIP eigenstates in the energy interval $[-0.1,0.1]$ for the case $L=150, U=1, V=1$, and $W=0.4$. The full line gives the best Breit-Wigner fit (13) with $\Gamma=0.0073$. The theoretical prediction is $\Gamma=0.0072$.

Fig. 3: Scaled Breit-Wigner width $\Gamma L / V$ as a function of the rescaled interaction $\frac{U}{4 V}$ computed in the energy interval $E / V \in[-0.1,0.1]$. The system size is $L=15$ (W/V = 1., empty circles), $L=25(W / V=1 .$, empty squares), $L=40(W / V=$ 0.6, empty diamonds), $L=60$ (W/V=0.5, full circles), $L=80(W / V=0.5$, full squares), $L=100(W / V=0.5$, full diamonds) $L=150(W / V=0.4$, full triangles up) and $L=200(W / V=0.35$, full triangles down $)$. The solid line gives the theoretical prediction (8), (9) multiplied by 2 to take symmetrization into account.

Fig. 4: Scaled Breit-Wigner width $\Gamma L / V$ as a function of the rescaled absolute value of the interaction $\frac{|U|}{4 V}$ computed in the energy interval $E / V \in[1 ., 1.2]$. The system size is $L=15(W / V=1$., empty circles), $L=25(W / V=1$., empty squares and $W / V=0.5$, empty diamonds), $L=40(W / V=0.8$, empty triangles up and $W / V=$ 0.5, empty triangles down), $L=60$ ( W/V $=0.5$, full circles), $L=80(W / V=0.5$, full squares), $L=100$ ( $W / V=0.5$, full diamonds), $L=100(W / V=0.5$, negative U, crosses), $L=150(W / V=0.4$, full triangles up), $L=200(W / V=0.35$, full triangles down) and $L=300(W / V=0.25$, full triangles left $)$. 

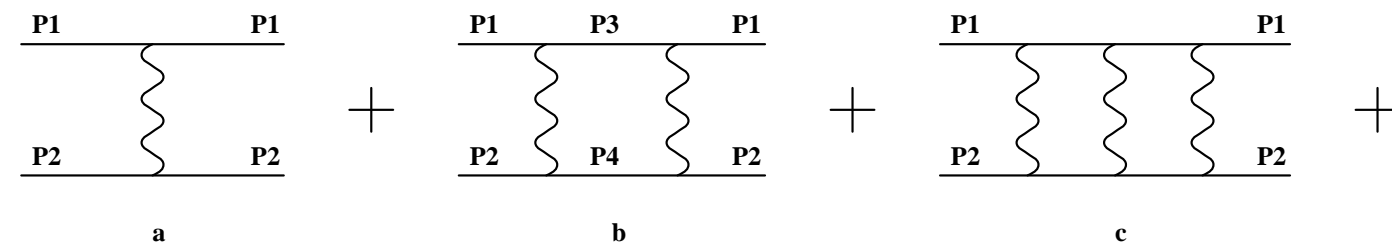

$+$

FIG. 1 


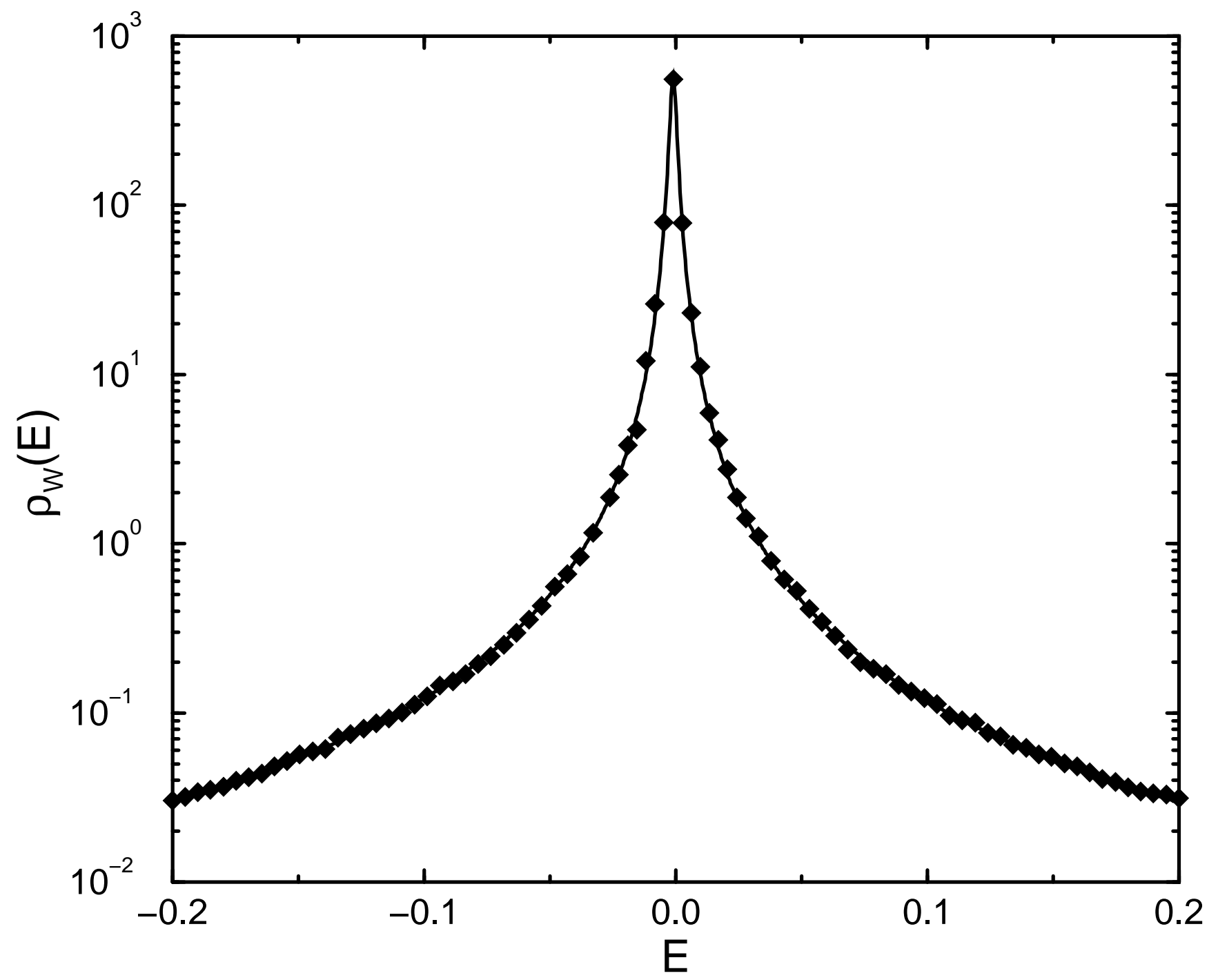




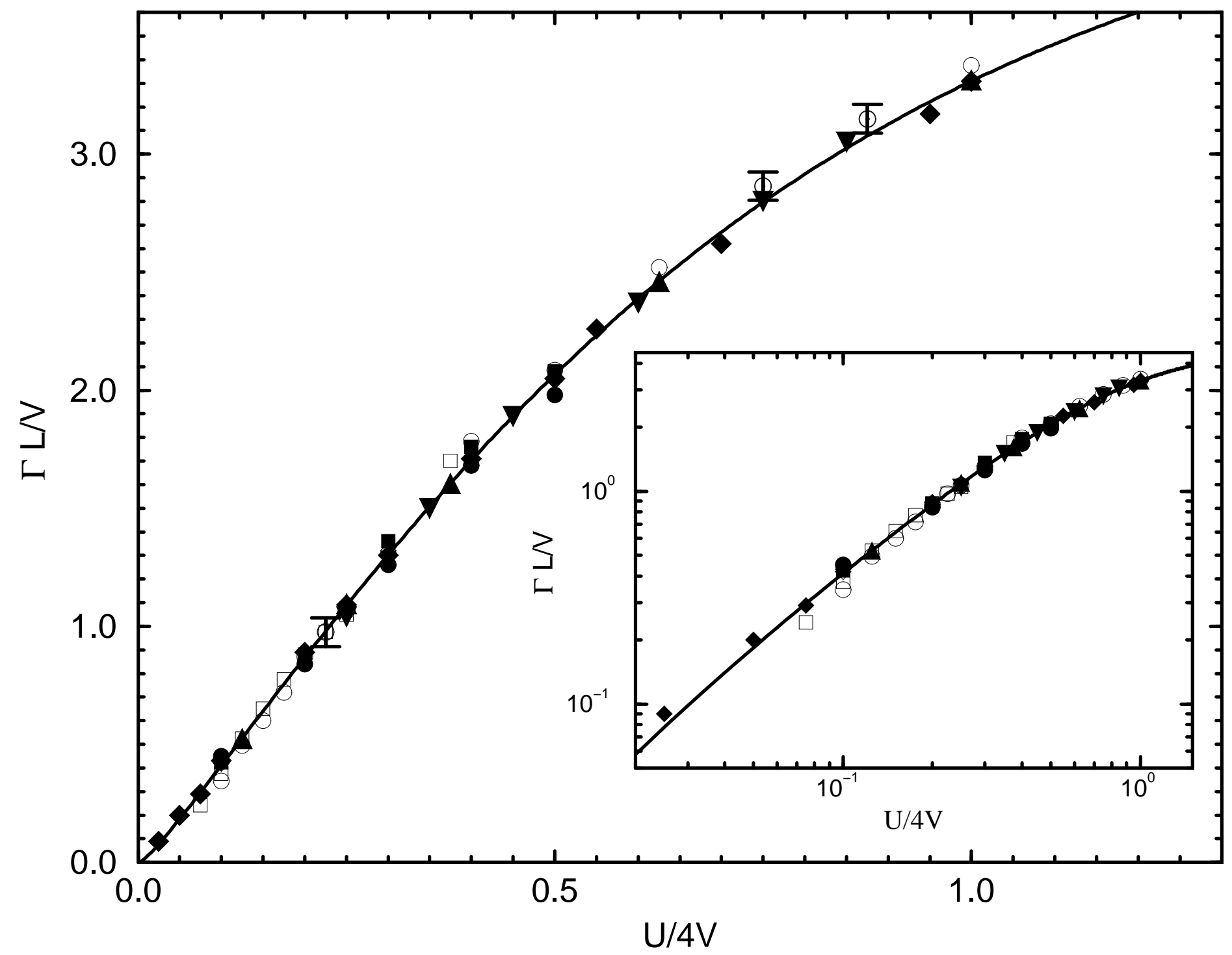




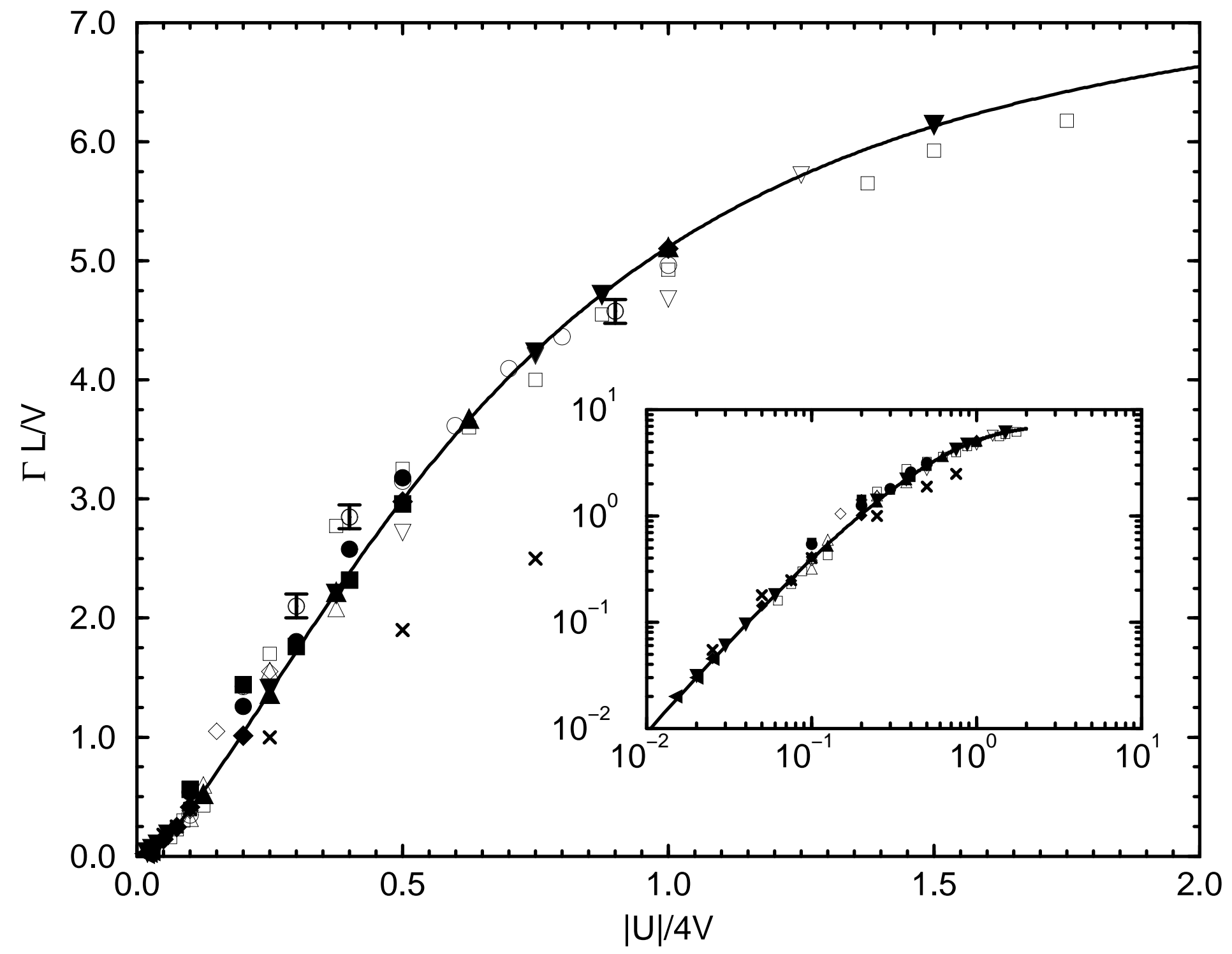

